\section{$\underset{\substack{\text { hommes } \\ \text { \& migrations }}}{ }$}

Hommes \& migrations

Revue française de référence sur les dynamiques

migratoires

1302 | 2013

Le Japon, pays d'immigration?

\title{
La participation politique des étrangers au Japon
}

\section{Yûji Nakano}

Traducteur : Pauline Cherrier

\section{CpenEdition}

Journals

Édition électronique

URL : http://journals.openedition.org/hommesmigrations/2486

DOI : 10.4000/hommesmigrations.2486

ISSN : 2262-3353

Éditeur

Musée national de l'histoire de l'immigration

Édition imprimée

Date de publication : 1 avril 2013

Pagination : 105-114

ISBN : 978-2-919040-22-3

ISSN : 1142-852X

Référence électronique

Yûji Nakano, «La participation politique des étrangers au Japon », Hommes \& migrations [En ligne],

1302 | 2013, mis en ligne le 31 décembre 2015, consulté le 03 mai 2019. URL : http://

journals.openedition.org/hommesmigrations/2486 ; DOI : 10.4000/hommesmigrations.2486 


\title{
LA PARTICIPATION POLITIQUE DES ÉTRANGERS AU JAPON
}

par YÛJI NAKANO, professeur de science politique, université Komazawa de Tokyo

\author{
Si le Japon contemporain est bien une société multiculturelle, \\ la vie politique y reste largement fermée à la participation \\ des étrangers. Mais la situation évolue au niveau local. Depuis \\ seize ans, la ville de Kawasaki, située entre Tokyo et Yokohama, \\ a mis en place un organisme consultatif destiné aux résidents \\ étrangers. Caisse de résonance de leurs revendications, espace \\ de dialogue et de propositions, cette assemblée qui représente \\ les citoyens étrangers entend améliorer leur insertion dans \\ la vie de la cité.
}

\section{Retour sur l'expérience de l'Assemblée représentative des citoyens étrangers de la municipalité de Kawasaki}

Dans le processus d'édification d'une société multiculturelle, quelle peut être la place de la participation des résidents étrangers à la vie publique, participation qui promeut leur reconnaissance et une répartition plus égalitaire des pouvoirs?

Le Japon s'est transformé en un véritable pays d'immigration suite à la réforme législative de 1989 qui permit l'ouverture des frontières aux nikkeijin (descendants de Japonais ${ }^{1}$ ). Cependant, le gouvernement japonais ne s'intéressait alors aux étrangers qu'en termes de politique du travail et de gestion de leur séjour. La question de l'intégration des résidents étrangers au Japon a donc tardé à être examinée au niveau national, ce débat n'ayant été abordé au sein d'un comité consultatif du ministère des Affaires étrangères quà partir de 2001. Le rapport qui en émana en 2004 insistait sur la nécessité de reconnaître les étrangers en tant que "membres à part entière de la sociétée”. Jusque-là, les communes où vivaient nombre d'habitants étrangers avaient, de leur propre initiative, développé des politiques à leur égard. En 2006, le ministère des Affaires intérieures et des Communications (sômushô), dont dépendent les autorités locales, s'appuya sur les consignes du rapport intitulé "Les étrangers en tant que résidents locaux", pour encourager l'élaboration d'un plan pour la promotion de la coexistence multiculturelle au sein des communautés locales 
(Plan for Multicultural Coexistence Promotion in Local Communities) devant servir de modèle aux départements et aux villes désignés par ordonnance gouvernementale ${ }^{3}$. Ce rapport servit de prélude à la multiplication de plans pour la promotion de la coexistence multiculturelle ${ }^{4}$ au sein des départements et des communes.

Des années 1995 jusqu'au

début des années 2000 , plusieurs

partis politiques ont soumis en vain à la Diète des propositions

de loi relatives à l'attribution

du droit de vote aux étrangers au niveau local, mais la Diète n'a jamais engagé de délibérations sérieuses à ce sujet.

"lélaboration de la coexistence multiculturelle au niveau local". C'est ce troisième pilier censé favoriser "la participation politique des résidents étrangers et la création de mécanismes permettant, grâce à des mesures prises au niveau des collectivités locales, de refléter leur opinion ${ }^{5 "}$ qui encouragea les collectivités locales à mettre en place un dispositif autorisant la participation des résidents étrangers à la vie publique.

Alors qu'une telle participation nécessite l'attribution du droit de vote aux étrangers, ce dernier n'est reconnu ni au niveau local, ni au niveau national. Des années 1995 jusqu'au début des années 2000, plusieurs partis politiques ont soumis en vain à la Diète des propositions de loi relatives à l'attribution du droit de vote aux étrangers au niveau local, mais la Diète n'a jamais engagé de délibérations sérieuses à ce sujet ${ }^{6}$. Le président des collectivités locales décida ainsi d'intégrer au lot de mesures prises par les localités à l'attention des étrangers des dispositifs de participation politique des résidents étrangers au niveau local, sous la forme de conseils consultatifs pour le développement de la coexistence multiculturelle. De tels dispositifs varient au sein des collectivités locales de par leur appellation, leur organisation et leur statut juridique. On parle aussi bien d'"assemblée des citoyens étrangers", que de "table ronde pour les citoyens de nationalité étrangère" ou encore de "conseil pour l'amélioration de la coexistence multiculturelle". Ces conseils sont parfois composés d'étrangers et de Japonais, parfois seulement d'étrangers (ceux ayant obtenu la nationalité japonaise étant ou non inclus). Les membres siègent à titre personnel ou au titre de leur statut académique ou associatif. En ce qui concerne le statut juridique, la plupart de ces conseils consultatifs s'appuient sur les yôkô ${ }^{7}$ (règlements intérieurs de l'administration) et, dans une minorité de cas, sur les "ordonnances locales" votées par les assemblées locales (départementales ou municipales). Les institutions créées par des "ordonnances locales" bénéficient d'un statut juridique plus stable que celles créées à partir de yôkô.

\section{L'histoire de l'Assemblée représentative des citoyens étrangers de la municipalité de Kawasaki}

En août 2012, seuls trois départements (Miyagi, Yamanashi et Shizuoka) et une vingtaine de communes disposaient d'organismes consultatifs permettant aux résidents étrangers et japonais de participer en qualité d'individus. Pour ce qui est

3. Sômushô (ministère des Affaires intérieures et des Communications (MAIC)), "Tabunka-kyôsei no suishin ni kansuru kenkyûkai-hôkoku, chiiki ni okeru tabunka-kyôsei no suishin ni mukete" ("Rapport d'activité du groupe de travail sur la promotion de la coexistence multiculturelle : vers une coexistence multiculturelle au niveau local”), 2006.4. Selon le ministère des Affaires intérieures et des Communications, "la coexistence multiculturelle au niveau local" se définit comme les circonstances dans lesquelles "les peuples de différentes nationalités et origines ethniques vivent ensemble en tant que membres de communautés locales, en respectant leurs différences culturelles et en s'efforçant d'y établir de bonnes relations" (MAIC, ibid., p. 5). 5. MAIC, "Tabunka-kyôsei no suishin ni kansuru kenkyûkai-hôkoku, chiiki ni okeru tabunka-kyôsei no suishin ni mukete", art. cit., p. 36. 6. Yûji Nakano, “Les propositions de loi sur le droit de vote des étrangers aux élections locales au Japon”, in Migrations Société, $n^{\circ} 83$, vol.14, 2002, pp. 9-16. 7. Un "yôkô" (règlement intérieur de l'administration) n'a pas de force légale en droit et est donc moins contraignant qu'une "ordonnance locale" (note de la traductrice). 8. L’article 94 de la Constitution du Japon attribue aux collectivités locales un pouvoir législatif autonome dans le cadre de la loi nationale. On utilise ici le terme d'“ordonnances locales" pour parler des lois votées par les collectivités locales (départementales ou municipales), afin de les différencier des arrêtés municipaux ou départementaux, qui sont des décisions administratives. Au niveau communal, ce n'est pas le maire qui établit les "ordonnances locales" mais l'assemblée municipale. 
des conseils composés uniquement de résidents étrangers, ils se trouvent dans les départements de Kanagawa et Aichi, et les communes de Saitama, Kawagoe, Kawasaki, Gifu, Toyohashi, Toyonaka, Okayama et Hiroshima. C'est aussi le cas de l'Assemblée représentative des citoyens étrangers de la municipalité de Kawasaki (ARCE) créée en 1996, l'un des premiers conseils consultatifs des résidents étrangers au Japon, mais surtout le seul établi par une ordonnance locale.

Lobjectif de cette assemblée n'est pas seulement de permettre la mise en place des mesures à l'attention des étrangers, mais aussi de compenser le fait qu'ils n'aient pas le droit de vote au niveau local. Il me semble ainsi important de présenter au lecteur français ce système de représentation des étrangers au niveau local, en tant qu'expérience pionnière au Japon, ainsi que ses résultats ${ }^{9}$.

À la fin de l'année 2011, la population de Kawasaki atteignait les 1430000 habitants, plaçant la ville en $8^{\text {e }}$ position au Japon, si l'on exclut les 23 arrondissements de la ville de Tokyo. La population étrangère de la ville s'élevait à 32000 habitants. Kawasaki est une ville portuaire située dans la zone industrielle entre Tokyo et Yokohama. Il s'agit d'une ville ayant historiquement accueilli de nombreux Coréens zainichi ${ }^{10}$. Ces Coréens ont d'ailleurs négocié durant de longues années avec les autorités municipales, notamment pour la suppression de la clause de nationalité qui empêchait les étrangers de bénéficier des allocations familiales et des logements sociaux. En outre, la ville de Kawasaki a développé ses propres mesures afin de compenser la relégation des étrangers hors du système national de protection sociale. Les Coréens zainichi de Kawasaki bénéficient donc depuis longtemps d'une

participation politique non institutionnalisée et ont fait l'objet de mesures favorables de la part des autorités municipales.

Vers la fin des années 1980, l'arrivée de nouvelles vagues de migrants à Kawasaki a rendu nécessaire la mise en place de mesures à l'attention non plus seulement des étrangers oldcomers mais aussi des newcomers ${ }^{11}$. La ville de Kawasaki commença à envisager le développement de ces mesures dès 1989. En 1993, un sondage d'opinion effectué auprès des étrangers de la ville confirma au maire de Kawasaki, Kiyoshi Takahashi, la ferme volonté de participation politique de ses résidents étrangers. Il déclara lors de l'assemblée municipale de mars 1994 : "Nous demandons au gouvernement d'accorder le droit de vote aux élections locales aux citoyens étrangers

L'objectif de cette assemblée n'est pas seulement de permettre la mise en place des mesures à l'attention des étrangers, mais aussi de compenser le fait qu'ils n'aient pas le droit de vote au niveau local.

(...). Pour compenser, nous souhaitons étudier les formes de participation politique des citoyens étrangers expérimentées dans d'autres pays au niveau municipal ${ }^{12}$." En se fondant sur les résultats de l'étude menée à partir des cas allemand et français, l'ordonnance municipale d'octobre 1996 institua l'ARCE ${ }^{13}$.

\section{La formation de l'Assemblée représentative}

L'ARCE prit la forme d'un organisme consultatif auprès du maire. Mais elle fut aussi créée afin de ressembler à un "système de substitution au droit de vote local". Le choix de cette appellation

\footnotetext{
9. L'auteur fait des recherches sur la participation politique des étrangers en France et au Japon. Il est membre depuis 2008 et président depuis 2010 du Comité consultatif relatif aux mesures pour la coexistence multiculturelle de la ville de Kawasaki. Ce comité a pour mission d'évaluer les mesures prises par la ville de Kawasaki, dont l'ARCE est un exemple, et d'émettre un avis au maire. Cet article est le résultat de plus de quinze ans d'observation de l'Assemblée et de quatre années de participation active. 10. Les Coréens zainichi (Coréens du Japon) sont les Coréens ayant immigré au Japon avant la Seconde Guerre mondiale et qui n'ont pas été rapatriés vers la Corée en 1945 . Ils étaient 600 ooo environ et perdirent leur nationalité japonaise en 1947. Ils sont aussi appelés immigrés “oldcomers" et ont longtemps formé le contingent d'étrangers le plus important du Japon (note de la traductrice). 11. Les termes de "oldcomers" et "newcomers", utilisés en anglais dans le monde académique, permettent de distinguer les migrants issus de la colonisation ou arrivés avant 1945 des migrants plus récents. Voir aussi l'introduction au dossier (note des coordinateurs). 12. Takao Yamada, "Kawasaki-shi gaikokujin-shimin daihyôsha-kaigi no seiritsu to genjô" (“La formation et la situation actuelle de l’Assemblée représentative des citoyens étrangers de la municipalité de Kawasaki”), in Takashi Miyajima (dir.), Gaikokujin shimin to seiji-sanka (Les Citoyens étrangers et la Participation politique), Tokyo, Yûshindô, 2000, p. 45. 13. "Kawasaki-shi gaikoku-jin shimin daihyôsha kaigi jôrei, jôrei 25-gô” (“Ordonnance n 25 du 3 octobre 1996 relative à l’Assemblée représentative des citoyens étrangers de la municipalité de Kawasaki”).
} 
d'“Assemblée représentative des citoyens étrangers" suggère d'emblée qu'il s'agit d'un système palliant le fait que les étrangers n'ont pas le droit de vote. Au moment de l'établissement de l'ARCE, la population étrangère de Kawasaki sélevait à un peu moins de 20000 habitants. L'assemblée est formée de 26 membres, nombre fixé en référence au code de l'autonomie locale ${ }^{14}$, qui limite à 26 le nombre des membres de l'assemblée municipale des communes dont la population est comprise entre 10000 et 20000 habitants $^{15}$.

Le mandat des représentants est de deux ans, renouvelable une fois. Les étrangers doivent, pour devenir représentants, remplir les conditions suivantes : être de nationalité étrangère, avoir au moins 18 ans et résider à Kawasa-

Les membres, nommés par le maire, doivent représenter proportionnellement les nationalités et le nombre d'étrangers présents

à Kawasaki. Leur sélection obéit aussi au respect des critères de parité des sexes et de lieu de résidence. des critères de parité des sexes et de lieu de résidence (il doit y avoir un équilibre entre les habitants du centre-ville et ceux des zones résidentielles) ${ }^{16}$. Au début de leur mandat, les représentants élisent à la fois le président et le vice-président de l'assemblée. L'ARCE est établie en tant que lieu de délibérations et de recherches faites par les résidents étrangers eux-mêmes sur des sujets concernant les résidents étrangers, considérés comme membres à part entière de la ville ${ }^{17}$. Les représentants choisissent leurs propres thématiques, souvent relatives aux mesures prises envers les étrangers, aux droits de l'homme, aux activités culturelles, aux associa- tions d'aide aux étrangers, mais surtout à tout ce qu'ils jugent nécessaire à la vie des étrangers. Une fois les délibérations terminées, ils soumettent au maire des propositions. Les représentants doivent incarner l'ensemble des résidents de la ville de Kawasaki, et non pas leur pays d'origine ou leur origine ethnique. Il fut ainsi décidé que le pays d'origine des représentants, de même que les problèmes internationaux, ne pouvaient pas faire l'objet de délibérations au sein de l'ARCE ${ }^{18}$. Le maire s'engage à respecter le contenu des propositions ${ }^{19}$ et doit présenter annuellement le rapport d'activité de l'ARCE à l'assemblée municipale.

Étant donné que les représentants ne sont pas élus par les résidents étrangers, il apparaît que l'ARCE ne fonctionne pas exactement comme une assemblée représentative. Néanmoins, le fait que les représentants ne sont pas élus au titre de leur nationalité ni des associations d'étrangers mais parlent au nom de l'ensemble des résidents étrangers de Kawasaki, comme le stipule l'ordonnance, ou encore le fait qu'ils forment un organe consultatif auprès du maire et peuvent décider librement de leurs thématiques de délibérations, nous permettent de souligner le rôle représentatif de l'ARCE, établie dans le but de pallier l'absence de droit de vote des étrangers.

\section{Bilan des seize années de l'Assemblée}

À chaque nouvelle session, l'ARCE commence par aborder les thèmes dont les représentants souhaitent débattre à partir de leur ressenti quotidien, de leur expérience de la discrimination ou de leurs activités bénévoles. Après une ou deux réunions, les thématiques sont définies et des groupes sont mis en place pour chacune d'elles. Les représentants disposent ensuite de deux ans (16 réu- 
Rue de Shin-Okubo où se concentrent des commerces coréens et chinois. @ Camille Millerand

nions au total) pour effectuer leurs recherches et leurs délibérations et, enfin, pour émettre des propositions. Afin de disposer d'informations et de connaissances suffisantes, l'ARCE peut demander des auditions auprès d'agents de la collectivité ou de personnes ressources extérieures à la collectivité en lien avec le sujet traité. Les propositions du rapport final présentées à la fin de chaque session (chaque année jusqu'à la $3^{\mathrm{e}}$ session qui a eu lieu d'avril 2000 à mars 2002) recouvrent une grande variété de mesures, dont certaines ont déjà été mises en œuvre. Nous présentons ci-dessous des exemples de propositions relevant des domaines de l'accès à l'information, de l'éducation et de la lutte contre les discriminations ${ }^{20}$.
Dans un premier temps, le plus important pour les étrangers est d'être en mesure de savoir où trouver, à la mairie et dans les mairies d'arrondissement, les informations relatives à la vie quotidienne mais aussi aux différentes procédures administratives et d'en comprendre le contenu. Dès la première année d'existence de l'ARCE, en 1996-1997, fut proposée "la mise en place de points d'informations pour les étrangers". En 1998, ces services d'informations pour étrangers distribuant des brochures en langue(s) étrangère(s) furent implantés dans les sept mairies d'arrondissement de la ville de Kawasaki, au sein des bibliothèques et des centres culturels. On rédigea aussi des brochures intitulées "À l'attention de tous les étrangers" comportant les informations et les coordonnées utiles aux étran- 
gers nouvellement arrivés à Kawasaki. Grâce à cet effort de traduction, les représentants ont instauré le multilinguisme à Kawasaki. Les brochures existent aujourd'hui en version papier mais sont aussi disponibles sur le site Internet de la ville en huit langues: japonais avec transcription phonétique, chinois, coréen, anglais, espagnol, portugais, tagalog et russe.

Dans les autres domaines liés à l'accès à l'information, les représentants préconisèrent les mesures suivantes : l'accompagnement de transcription phonétique pour les documents rédigés en japonais et distribués dans les écoles, la publication d'informations sur les logements sociaux pour les résidents étrangers, la mise en place d'un système de transmission d'informations en cas de catastrophe naturelle, la mise à disposition d'une liste des hôpitaux disposant de médecins parlant des langues étrangères, etc. Aujourd'hui, le "plan d'évacuation d'urgence" est disponible en sept langues, et il est désormais possible de trouver sur le site Internet de la ville de Kawasaki la liste des services médicaux où exercent des médecins parlant des langues étrangères.

L'éducation des enfants est également une préoccupation de premier ordre pour les résidents étrangers. Les représentants ont recommandé à plusieurs reprises (en 1996-1997, 2003-2004, 2011-2012) que les écoles puissent offrir aux enfants, étrangers comme japonais, une éducation leur permettant de découvrir la diversité culturelle constitutive du monde. En 2001-2002, ils préconisèrent aussi un soutien scolaire pour les enfants étrangers scolarisés dans les écoles japonaises, en particulier pour ceux qui ne maîtrisent pas la langue japonaise. Au Japon, l'entrée au lycée est conditionnée par la réussite à un examen, or les cours privés préparatoires à cet examen représentent un obstacle pour les enfants étrangers. Les représentants demandèrent donc en 2007-2008 un soutien scolaire pour aider les élèves étrangers à préparer l'examen d'entrée au lycée, mais aussi la mise en place d'un système d'examen d'entrée spécifique pour les étrangers. Le département de Kanagawa, où se trouve Kawasaki, dispose déjà dans une autre ville de lycées départementaux avec une procédure d'entrée spécifique pour les étrangers. Cependant, les nombreuses requêtes des représentants pour la création d'un tel lycée municipal à Kawasaki n'ont jamais pu aboutir pour des raisons financières.

Les écoles de Kawasaki emploient depuis 1999 des enseignants étrangers, les élèves japonais ayant désormais la possibilité de côtoyer de près une culture différente de la leur. Les élèves étrangers dont le niveau de japonais est insuffisant bénéficient pendant six mois de la présence deux fois par semaine d'enseignants de japonais qui parlent leur langue maternelle : ils peuvent ainsi mieux les aider dans leur apprentissage du japonais et leur apportent un soutien psychologique. Les représentants ont réclamé à de nombreuses reprises l'amélioration de ce système et de son efficacité.

\section{L'abolition des discriminations}

L'une des questions prioritaires pour les représentants est d'éliminer les discriminations à l'encontre des résidents étrangers. Comme on l'a vu précédemment, les oldcomers coréens zainichi avaient déjà exigé de la municipalité l'abolition de pratiques discriminatoires. En réalité, bien avant la mise en place de l'ARCE, la clause de nationalité pour l'entrée dans les logements sociaux avait été supprimée et les résidents étrangers ont réussi à bénéficier de l'allocation municipale pour enfant, réservée auparavant aux Japonais.

Au début de la création de l'ARCE, la discrimination au logement dans le parc locatif privé était encore très répandue. Il est pourtant difficile de différencier physiquement les étrangers oldcomers des Japonais. Pourtant, au moment de la rédaction du 
contrat de location, il n'est pas rare que le propriétaire le fasse annuler s'il découvre que les futurs locataires sont coréens, même si leurs moyens financiers sont suffisants. Nombreux sont les propriétaires qui ne souhaitent pas non plus louer leur appartement aux newcomers qui n'ont pas la réputation de faire du bruit lorsqu'ils organisent des fêtes chez eux ou de ne pas respecter les règles concernant le tri des ordures.

De telles expériences ont incité les représentants à proposer, dès le début du fonctionnement de l'ARCE, en 1996-1997, une "ordonnance sur le logement" interdisant la discrimination contre les étrangers. La proposition étudiée par le comité de consultation pour le logement déboucha sur la promulgation d'une ordonnance fondamentale sur le logement de la ville de Kawasaki, entrée en vigueur en avril 2000. Le premier alinéa de l'article 14 de cette ordonnance stipule: "Aucun individu ne doit se voir refuser sans raison légitime l'accès à un logement en location privée ni à une résidence stable, pour cause de son âge, d'un handicap, de son origine, etc." Lordonnance interdit la discrimination pour l'accès au logement, non seulement pour les étrangers mais aussi pour d'autres catégories de personnes. Elle définit en outre à l'alinéa 2 du même article les conditions selon lesquelles le maire peut demander aux parties concernées de coopérer et d'améliorer la situation en cas de refus de location ou d'accès à un lieu de résidence stable. L'ordonnance fondamentale sur le logement de la ville de Kawasaki se caractérise donc par la possibilité pour le maire d'intervenir activement.

La location d'un appartement au Japon requiert généralement le soutien d'un garant, le plus souvent un parent ou une connaissance proche. Ce qui paraît difficile pour des étrangers qui viennent d'arriver au Japon. En 1997-1998, l'ARCE recommanda la mise en place d'un système public de garant. Aujourd'hui, la ville de Kawasaki peut présenter aux étrangers des sociétés qui se portent garantes pour eux lors de la recherche d'une location. Malgré ces mesures, les représentants continuent de ressentir des discriminations dans l'accès au logement. Ils ont donc soumis de nouvelles propositions en vue de résoudre le problème.

\section{Améliorer la participation des étrangers à la vie publique}

Le second exemple concernant la lutte contre les discriminations porte sur la clause de nationalité interdisant l'accès aux emplois de la fonction publique aux étrangers. En effet, selon la loi, un certain nombre de postes dans la fonction publique font l'objet d'une clause de nationalité (diplomate, membre de la commission électorale, etc.), par contre, pour tous les autres postes de fonctionnaire, la loi ne précise rien. Malgré l'absence de définition juridique sur la question, le gouvernement japonais s'est prononcé en 1953, déclarant : “La nationalité japonaise est un prérequis pour tout poste de la fonction publique relevant de l'exercice du pouvoir public ou participant à la construction nationale", exigeant ainsi que la nationalité japonaise soit obligatoire pour L'ordonnance municipale d'avril 2009 établit à Kawasaki le référendum local, dispositif permettant de refléter les diverses opinions des citoyens. les fonctionnaires des administrations locales. Suite à la mise en place de l'ARCE en mai 1996, la ville de Kawasaki examina la description et le contenu des postes et jugea que, à l'exception du métier de pompier, aucun emploi n'était concerné par "lexercice du pouvoir public" ou "la participation à la construction nationale", supprimant alors la clause de nationalité. Cette suppression reste cependant partielle, les étrangers ne pouvant pas être promus à un poste de direction. Cette limite n'a toujours pas été abrogée malgré la proposition des représentants en 1999-2000 d'une "abolition totale de la clause de nationalité" afin de permettre la promotion professionnelle des étrangers à des postes de direction. L'ARCE exige aussi, en tant que "système de substitution au droit de vote des étrangers", l'institution du droit de vote des étrangers 


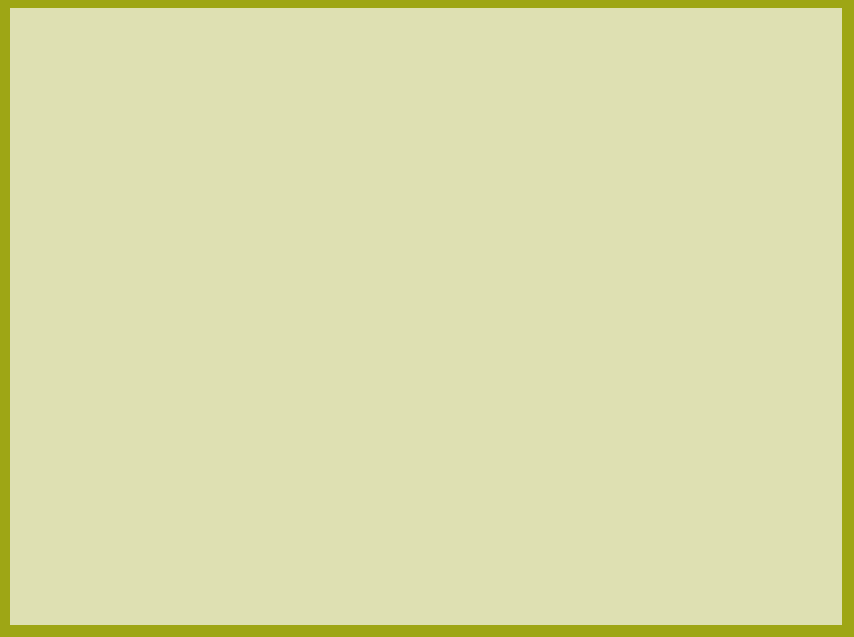

Séance mensuelle de l'Assemblée représentative des citoyens étrangers de la municipalité de Kawasaki. (c) MAIRIE DE KAWASAKI

aux élections locales, droit qui ne peut être obtenu sans faire l'objet d'une loi adoptée par la Diète. Les représentants ont ainsi recommandé en 20052006 à la ville de Kawasaki de faire appel au gouvernement pour la mise en œuvre du droit de vote des étrangers aux élections locales.L'ordonnance municipale d'avril 2009 Durant ces seize années établit à Kawasaki le réféd'expérience, les résidents rendum local, dispositif étrangers de Kawasaki permettant de refléter les ont pu exprimer leurs opinions

concernant les politiques locales et la municipalité a pris l'habitude de les prendre en compte. tementales et municipales, du maire et du gouverneur du département, toutefois, les collectivités locales peuvent librement se doter de systèmes électoraux propres tels que le référendum local ${ }^{21}$. Ainsi, en 2002, le système de vote de Kawasaki a été mis à l'étude et les membres de l'ARCE ont pro- posé en 2003-2004 que la participation des résidents étrangers soit incluse dans le plan d'élaboration de la nouvelle ordonnance sur le référendum local.

Le système actuellement mis en place intègre la proposition de l'ARCE et permet aux étrangers âgés de 18 ans révolus, résidant depuis trois ans minimum au Japon et trois mois au moins dans la ville de Kawasaki, de participer aux référendums locaux. Mais aucun référendum n’a été organisé jusqu'à aujourd'hui. Nous ne pouvons pas présenter ici toutes les propositions de l'ARCE mais, comme nous l'avons déjà évoqué, la municipalité tente de répondre à ses diverses demandes en élaborant de nouvelles politiques. Durant ces seize années d'expérience, les résidents étrangers de Kawasaki ont pu exprimer leurs opinions concernant les politiques locales et la municipalité a pris l'habitude de les prendre en compte.

\section{Les difficultés de l'Assemblée représentative}

Au fil des propositions faites depuis seize ans, on peut considérer que la ville de Kawasaki a mis en place des mesures favorables aux étrangers et a œuvré à la réalisation de la coexistence multiculturelle. Néanmoins, l'accumulation de ces propositions a eu des effets négatifs au sein de l'ARCE.

Aux premiers temps de l'Assemblée, le contenu des propositions paraissait inédit et il était aisé pour la ville de mettre en place de nouvelles mesures, comme l'ordonnance municipale pour le logement et le service d'information pour les étrangers. En 1999, le président de l'ARCE exprima sa grande 
satisfaction quant à la rapidité avec laquelle les autorités municipales réagirent aux propositions de l'ARCE entre 1996 et 2000, entérinant ainsi son utilité2 ${ }^{2}$. Mais, au fur et à mesure, en raison de l'aspect redondant des thématiques dont souhaitaient débattre les représentants et de l'allongement croissant du temps consacré aux délibérations, l'ajournement du traitement de certaines propositions est devenu fréquent. Lorsque l'on lit les comptes rendus des délibérations, on observe une certaine difficulté à différencier les propositions passées des propositions nouvelles, surtout à propos des sujets qui préoccupent le plus les représentants, à savoir la manière de procurer des informations aux étrangers et l'éducation à l'interculturalité.

Cette répétition des thématiques au sein de l'ARCE a entraîné une insatisfaction envers la municipalité et exercé une mauvaise influence sur les représentants qui se sont mis à douter de l'utilité de leur travail. Les autorités municipales classent les propositions selon un ordre d'état d'avancement ( $A=$ résolues, $B=$ en cours de réalisation, et $C=$ pas encore abordées). Dans de nombreux cas, comme nous venons de l'évoquer, les représentants souhaitent poursuivre le débat pour des propositions déjà faites dans le passé, considérant que leur contenu pose encore problème. Or, lorsque les représentants souhaitent se saisir d'une thématique et qu'il s'agit d'une proposition abordée antérieurement, les autorités municipales l'ont déjà évaluée comme A, alors qu'elle reste problématique pour les représentants. Le sentiment de satisfaction des représentants n'est donc aujourd'hui plus du tout le même qu'aux débuts du fonctionnement de l'ARCE.

Il faudrait, avant tout, que les représentants aient une juste compréhension des efforts déjà déployés par la municipalité pour tenir compte des propositions faites par le passé. Or le contenu des délibérations devient inéluctablement plus complexe au fur et à mesure que le temps passe. En réalité, pour les nombreux représentants dont le japonais n'est pas la langue maternelle, il n'est pas aisé de com-

prendre, en langue japonaise, l'accumulation progressive des propositions et les efforts déployés par la municipalité pour les intégrer. En outre, même lorsqu'elles font l'objet de propositions par l'ARCE, les délibérations portant sur des thématiques qui ne relèvent pas toujours de la compétence de la ville ne peuvent faire l'objet de requêtes qu'auprès des autorités compétentes au niveau départemental ou national. Cette répartition des compétences des pouvoirs publics étant déjà difficile à saisir pour les Japonais, elle l'est encore bien davantage pour les résidents étrangers.

Il est possible qu'en début de mandat, les représentants, encore peu informés du travail accompli par la

En réalité, pour les nombreux représentants dont le japonais n'est pas la langue maternelle, il n'est pas aisé de comprendre, en langue japonaise, l'accumulation progressive des propositions et les efforts déployés par la municipalité pour les intégrer.

municipalité en faveur des étrangers, ressentent une certaine frustration. Il se pourrait aussi qu'ils soupçonnent d'indifférence la municipalité lorsqu'ils s'attellent à résoudre un problème dont le traitement ne relève pas de la compétence de la ville. Ces frustrations potentielles disparaissent progressivement au long des deux années de leur mandat, au cours desquelles l'expérience des délibérations et les explications apportées par les agents de collectivité leur permettent de comprendre le fonctionnement et la répartition des compétences au sein des collectivités locales. L'ARCE permet donc aux résidents étrangers de recevoir une certaine éducation politique, ce qui joue un rôle significatif comme dispositif de substitution au droit de vote des étrangers. C'est pourquoi il est important que les représentants soient renouvelés tous les deux ans, afin qu'un maximum d'étrangers puissent bénéficier de cette expérience. Pour que l'ARCE lance des propositions efficaces et que son rôle ne soit pas limité à la simple participation des résidents étrangers ayant un effet éducatif et politique, il conviendrait de résoudre le problème de l'accumulation des propositions. La plupart des 
résidents, dont les étrangers éligibles comme représentants, n'ont pas de connaissances préalables sur les propositions précédentes, ni même sur les efforts déployés par la municipalité, et il est à présent rare que, au cours des deux années ou des 16 sessions de délibérations de l'Assemblée, des propositions inédites soient faites. Dans ce contexte, il apparaît important, afin d'élaborer des propositions efficaces, de créer une forme de conseil auquel participeraient les représentants qui possèdent une expérience plus longue de l'ARCE. Ou encore, on pourrait imaginer un moyen qui permette aux représentants d'évaluer eux-mêmes l'état d'avancement des mesures résultant des précédentes recommandations. On pourrait soit changer la manière actuelle de mener les délibérations en présentant une thématique à débattre à partir de l'expérience personnelle des représentants pour ensuite la restreindre, soit créer une instance séparée pour évaluer les résultats des politiques.

\section{Conclusion}

Dans la ville de Kawasaki s'est mis en place un processus d'élaboration de mesures politiques fondé sur la prise en compte des propositions d'une Assemblée représentative des résidents étrangers. Ce mécanisme a permis aux résidents étrangers de participer à la vie politique de la ville. Toutefois, les seize années d'activité, plus précisément l'accumulation de seize années de propositions et de réponses politiques faites par la ville, ont débouché sur l'insatisfaction croissante des représentants étrangers à l'égard de la municipalité et sur la remise en question de la raison d'être de l'Assemblée représentative elle-même. Cela entraînant de plus en plus de difficultés à recruter de nouveaux représentants.

L'Assemblée fait face aujourd'hui à de sérieux problèmes et plusieurs mesures pourraient être prises afin d'assurer son avenir. Je souhaite avancer deux propositions. Premièrement, la municipalité ayant rassemblé sous la forme d'un fascicule l'ensemble des propositions et des réponses politiques faites au cours des seize années d'activité, il faudrait que ce document soit traduit en plusieurs langues. Les résidents étrangers, dont la langue maternelle n'est pas le japonais, pourraient ainsi maîtriser l'ensemble des débats. Cela permettrait aussi de motiver plus facilement de nouveaux candidats. Le second point concerne les évaluations par les représentants étrangers des réponses politiques apportées à leurs demandes. Il est nécessaire de réduire le décalage entre leurs attentes et celles des services municipaux concernant l'ampleur des mesures prises pour répondre aux demandes de l'Assemblée, cela afin de tempérer les sentiments d'insatisfaction à l'égard de la municipalitée ${ }^{23}$.

L'ARCE de Kawasaki, créée par ordonnance municipale, a été une initiative pionnière au Japon qui a mis en place des dispositifs novateurs assurant la participation politique des résidents étrangers. Dans le souci de valoriser cette participation politique, il serait bon que la résolution des difficultés actuelles de l'Assemblée puisse aussi venir de ses membres.

Traduit du japonais par Pauline Cherrier, maître de conférences, université d'Aix-en-Provence. 